\title{
II.
}

\section{Ueber hyaline Entartungen in den Lymphdrüsen.}

\author{
Aus dem pathologischen Institute von Herrn Prof. von Recklinghausen
} zu Strassşurg.

Von Leo Wieger, Cand. med.

(Hierzu Taf. 1.)

Schon seit längerer Zeit war Herrn Professor von Recklinghausen gelegentlich, als zufälliger Befund bei Sectionen, eine eigenthümliche Entartung der Lymphdrüsen aufgefallen. Dieselben zeigten auf dem Schnitte weissliche, netzformig angeordnete Zeichnungen. Bei der mikroskopischen Untersuchung liessen sich die Netze auflösen in Züge von theils verkalkten, theils eigenthümlich hyalinen Gefässen. Auf Anregung von Herrn Professor von Recklinghausen habe ich versucht diese Affection näher zu verfolgen. Im Verlaufe von neun Monaten gelang es mir, in dem pathologischen Institute der hiesigen Universitât, bei einer Zahl von circa 600 Sectionen, dieselbe zehn Mal zur Untersuchung zu bekommen. Die Resultate dieser Untersuchung sind in vorliegender Arbeit zusammengestellt.

Technisch wurde folgendermassen verfabren. Von den frischen Lymphdrüsen wurden feine Scbnitte entnommen. Als Zusatzflüssigkeit diente eine $\frac{3}{4}$ procentige Kochsalzlösung. Ein Theil der Drüsen wurde zur Härtung in Alkohol oder Chromsäure eingesetzt. Alkohol leistete zu diesem Zwecke, bei der meist schon ziemlich beträchtlichen Consistenz der kleinen Organe, stets sehr gute Dienste. Dasselbe Lob lässt sich der Chromsäure nicht zollen. Wenn sie auch den Zusammenhang der Elemente mehr schonte, so war sie doch öfters durch sehr unangenehme Missstände recht nachtheilig. Es sei zum Beispiel nur der Schwierigkeit der Färbungen, und der gelegentlichen gründlichen Beseitigung von Prozessen wie Verkalkung gedacht. Die von gehärteten Drüsen entnommenen Schnitte wurden theils ungefärbt, theils nach möglichst sorgfältiger Färbung, 

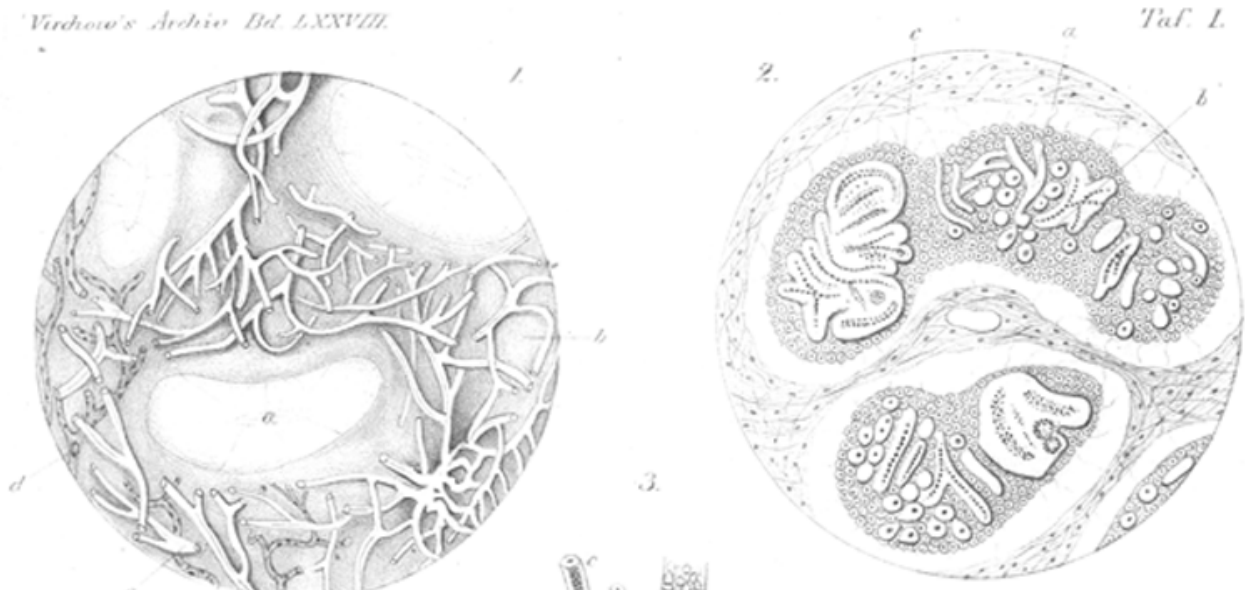

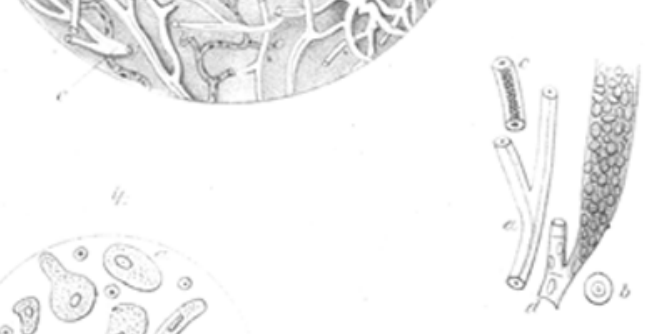
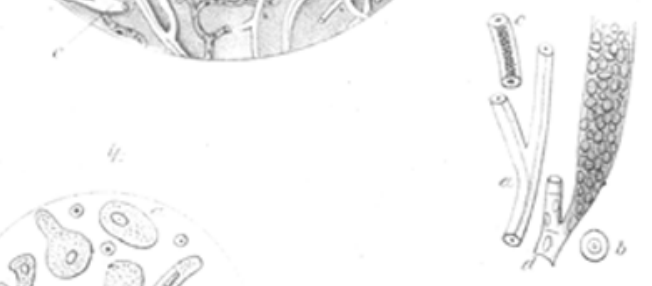

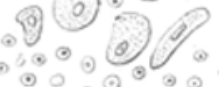

${ }^{\circ} \odot \odot \odot^{\circ} \odot \stackrel{\circ}{\circ} \odot$

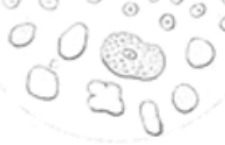
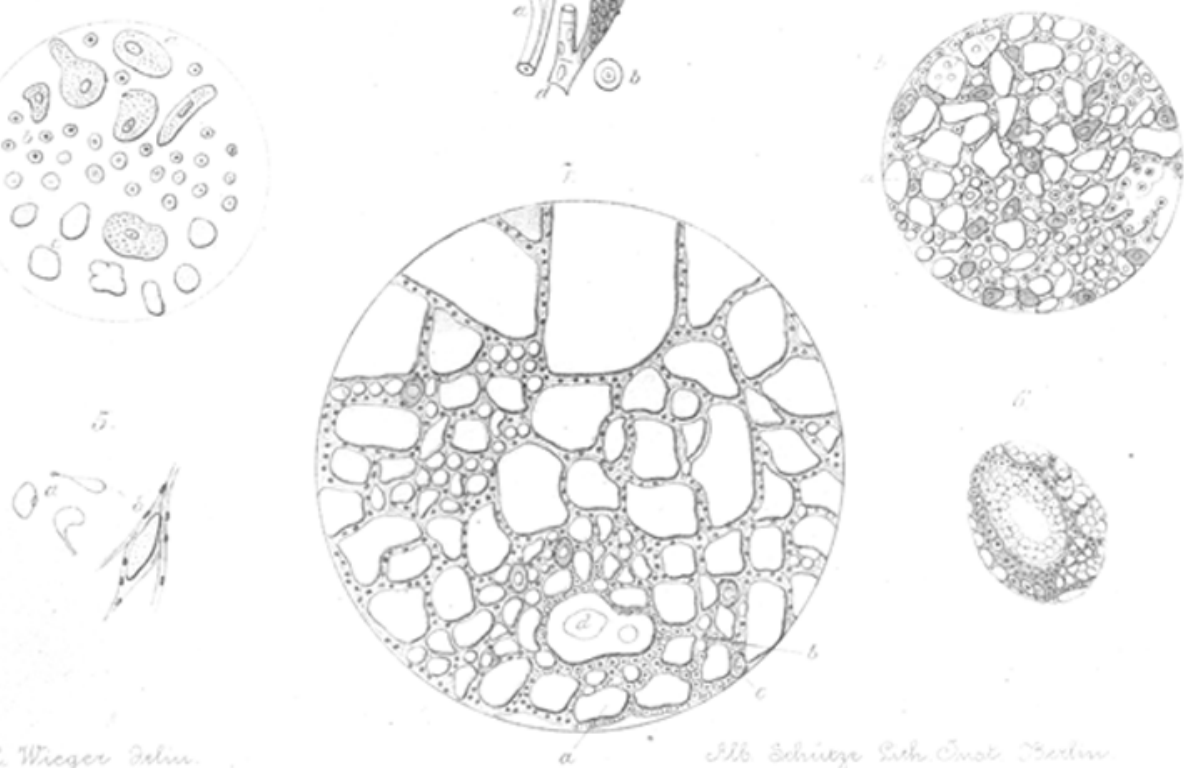

9. Wheger atwes

a 
bei Glycerinzusatz untersucht. Von den verschiedenen Färbemitteln seien hier besonders bervorgehoben Hämatoxylin, Anilinbraun, Pikrocarmin, Eosin in Verbindung mit Hämatoxylin. Als mechanische Hülfsmittel wurden Ausschütteln und Auspinseln angewandt. Die Präparate wurden ausschliesslich in Glycerin untersucht. Lack erwies sich als untauglich, ausser bei Uebersichtspräparaten. Die bei weitem anschaulichsten Bilder lieferten aber stets frische Schnitte, besonder's der grösseren Dicke wegen, welche die Durchsichtigkeit des frischen Gewebes den Schnitten zu geben erlaubte.

\section{A. Hyaline Entartung der Lymphdrüsengefässe.}

Bei dieser Affection zeigt der äussere Habitus der Drüsen, ausser einer ziemlich constanten, gewöhnlich mässigen, seltener beträchtlichen Schwellung, gar nichts Charakteristisches. Die Consistenz ist gewöhnlich normal, seltener fest, selbst derb. Die Drüsenkapsel erscheint meist von fleischig rother Farbe, ziemlich stark vascularisirt und injicirt. Selten, und nur bei sehr bejahrten Individuen sind die Drüsen blass, von mehr fibröser Consistenz.

Auf dem Schnitte dagegen bieten sie ein constantes, typisches, makroskopisch leicht erkennbares Bild dar. Statt des normalerweise ziemlich gleichmässig körnigen, mässig injicirten, homogen fleischrothen, etwas auf dem Schnitte vorquellenden Parenchyms, mit den eingesprengten, etwas einsinkenden Trabekeln, erscheint ein ziemlich stark, selbst sehr stark blutreiches Gewebe, welches durch ein äusserst feines und zierliches Netz von weisslichen, mehr oder weniger opaken Bälkchen, in eine grosse Anzahl von kleinen Feldern zerlegt wird. Die Felder bieten im Allgemeinen makroskopisch die Charaktere eines normalen, etwas schlaffen und hyperämischen Lymphgewebes dar. Sie sinken auf dem Schnitte leicht ein, die Bälkchen dagegen springen etwas vor. Beim Betasten fühlen sich die Bälkchen derb, selbst sandig rauh an. An den Stellen, wo mehrere Bälkchen sich scbneiden, erscheinen besonders derbe Knotenpunkte. Diese ganze, höchst zierliehe Zeichnung kommt vorzugsweise in der Rindenschicht der Drüsen zur höchsten Ausbildung. In der Marksubstanz dagegen zeigen sich nur einzelne derbere, mehr 
lineäre Bälkchen. In den seltenen Fällen, wo, wie erwähnt, die Drüsen mehr blass fibrös erscheinen, lässt sich diese Zeichnung nicht bemerken, wahrscheinlich weil sie bei der allgemeinen Derbheit und Blässe des Gewebes nicht hervorsticht. Es ist in diesen Fällen dann makroskopisch ein Schluss auf Anwesenheit des Prozesses nicht wobl möglich. Doch dürfte gelegentlich die sandige Rauhigkeit der Schnittfläche die Aufmerksamkeit erwecken.

Durchmustert man nun, in einern gegebenen Falle, sämmtliche Lymphdrüsengruppen der betreffenden Leiche, so ergeben sich bald in Betreff der Vertheilung der Affection interessante Resultate. Der Prozess ist kein für den gesammten Lymphdrüsenapparat allgemeiner und gleichmässiger. Einzelne Gruppen bleiben vollständig verschont, mit anderen verhält es sich in den einzelnen Fällen sehr verschieden. Selbst in den afficirten Gruppen sind die einzelnen Drüsen ungleich stark ergriffen. Jedoch ergab sich in einer Reihe vollständig untersuchter Fälle eine gewisse Regelmässigkeit, eỉne gewisse Reihenfolge in dem Ergriffenwerden der Drüsenapparate. Dasselbe erfolgt stets gruppenweise. Die erste, constant am intensivsten ergriffene Gruppe ist die der Lumbaldrüsen. Bei einer Prüfung der Leichen auf ein eventuelles Vorhandensein der Affection, konnte man es stets bei der Untersuchung der Lumbaldrüsen bewenden lassen. Waren dieselben nicht afficirt, so liess sich auch die Affection an den übrigen Lymphdrüsen des Körpers nicht nachweisen. Es folgten auf die Lumbaldrüsen die Inguinaldrüsen, dann seltener die Axillardrüsen, sehr selten die Jugulardrüsen. Die Mesenterialdrüsen zeigten die Affection niemals. Die Bronchialdrüsen zeigten meist nur die gewöhnliche fibrös-schiefrige Entartung, was übrigens bei dem durcbschnittlich hoben Alter der Individuen auf Besonderheit keinen Anspruch macht. Gewöhnlich bilden aber innerhalb jeder einzelnen Drüsengruppe diejenigen, welche die Affection aufweisen, die Mehrzahl. In sehr hochgradigen Fällen bleiben selten Drüsen in namhafter Anzahl verschont.

Bei der mikroskopischen Untersuchung zeigt das noch vorhandene Parenchym im Grossen und Ganzen die Charaktere eines normalen Lymphgewebes, die reticuläre Substanz ist zart, feinfaserig, die Kapsel und die Septen, welche von ihr gegen die Marksubstanz eindringen, zeigen nichts Abnormes in ihrem Bau und Verhalten, die Differenzirung zwischen Follikel und Lymphsinus ist, wie ja 
schon normalerweise, je nach den einzelnen Drüsen, in verschiedenem Grade deutlich, im Allgemeinen aber leicht zu bewerkstelligen, die Lymphbahnen erscheinen im Allgemeinen normal. In der parenchymatösen Substanz eingebettet liegen nun die beschriebenen Bälkchen. Dieselben lassen sich auflösen in Züge von cylindrischen Gebilden, welche ausschliesslich in der Follicularsubstanz gelagert, in Gestalt reichlich dichotomisch verästelter, an Caliber allmählig abnebmender Stränge erscheinen. Niemals erscheinen diese Gebilde in den Lymphbahnen selbst, oder in nächster Nähe derselben, sondern siê bleiben von ihnen stets durch eine, wenn auch gelegentlich dünne Schicht lymphatischen Drüsengewebes getrennt. Ist die Entartung sehr weit vorgeschritten, so bilden sie sehr dichte Netzfiguren, oder sie ballen sich zu Knäueln zusammen, welche gelegentlich den grössten Theil der Drüse ausmachen können.

Mit Rücksicht auf diese Anordnung lässt sich mit Wahrscheinlichkeit schliessen, dass es sich hierbei um Blutgefässe handeln dürte. Es wird diese Annahme dadurch bestätigt, dass eine grosse Anzahl dieser cylindrischen Gebilde mit einem Lumen versehen sind, dass sie also Kanäle darstellen. Die Anzahl dieser Gebilde lässt sie aber nur für capilläre Gefässe halten. Damit dieselben aber solche Dimensionen zeigen können, muss die Volumzunahme, welche an ihnen vor sich gegangen ist, eine recht beträchtliche sein. Die geringsten Aestchen zeigen durchschnittlich einen Durchmesser von $0,03 \mathrm{Mm}$. Dass diese Volumzunahme nicht auf einer einfachen Dilatation des Gefässkanals beruht, ergiebt sich bei einfacher Betrachtung sogleich. Der Gefässkanal erscheint nämlich bei Längsansicht des Gefässes als eine, im Verhältniss zur Gesammtdicke des Gefässes sehr dünne Axenlinie; stellenweise ist sogar der Gefässkanal bis zur Undeutlichkeit verengt und scheint selbst gelegentlich ganz obliterirt zu sein.

Noch auffallender aber als die Volumzunahme der Gefässe ist die Beschaffenheit des Materials, durch welches diese Volumzunahme zu Stande kommt. Dasselbe ist sehr diaphan, glänzend, sehr stark lichtbrechend, im Ganzen sehr ähnlich der Grundsubstanz des hyalinen Knorpels. Die Begrenzung dieser Substanz nach aussen ist gewöhnlich scharf, nicht aber stets geradlinig, sondern oft leicht sinuös und buchtig, indem die Dicke des Gefässrohres stellenweise wechselt. In welchem Verhältnisse der hyaline Mantel zu den nor- 
malen Gefässhäuten steht, wird am besten an Querschnitten deutlich. Diese sind in den Präparaten in ziemlicher Anzahl vorhanden, in Gestalt von grösseren und kleineren, kreisförmigen, scharf contourirten Scheiben. Diese Scheiben sind aus hyaliner, stark lichtbrechender Substanz gebildet. Sie zeigen im Centrum ein rundes oder seltener leicht faltiges, gewöhnlich aber sehr enges Lumen. Sehr selten, und nur in solchen Fällen, wo der Prozess weiter vorgeschritten ist, kommt es vor, dass ein Lumen kaum mehr angedeutet, oder gar nicht mehr vorhanden ist; das ganze Scheibchen ist dann gleichmässig homogen hyalin. Diejenigen Querschnitte nun, welche noch ein Lumen aufweisen, lassen hie und $d a$ in demselben kleine Kerne und Körner erkennen. Diese sind allem Anscheine nach als eine in sehr atrophischem Zustande befindliche Intima zu betrachten. Hie und da schien es, allerdings ziemlich selten, als könne erstens eine solche Intima in Resten, zweitens eine darauffolgende Zone unterschieden werden, welche zwar von den gewöhnlichen Elementen einer Gefässmedia nichts mehr erkennen liess, aber doch Kalkkörner und Fettropfen nebst albuminösem Detritus zeigte, somit Producte abgelaufener regressiver Metamorphosen, wie sie namentlich auch in der mittleren Haut von Arterien vorkommen. Der hyaline Mantel lag dagegen stets erst ausserhalb dieser Zone, also an Stelle der normalen Membrana adventitia der Gefässchen, in Gestalt eines glashellen, absolut amorphen Ringes. Selbst die sorgfältigsten Tinctionen und Behandlungen mit Reagentien konnten an ihm Kerne, oder irgend etwas was mit Zellterritorien Aehnlichkeit hätte, nicht zu Gesicht bringen. Nur in seltenen Fällen, und zwar stets an Chromsäurepräparaten, schien es als zeige die byaline Substanz eine Menge feinster Linien in concentrischer Anordnung. Dieše Linien liessen sich aber niemals genauer einstellen. Sie waren wahrscheinlich nur der optische Ausdruck für Faltungen der Substanz, welche dem erhärtenden Agens zugeschrieben werden dürften. Aehnliches wurde an in Alkohol gehärteten Präparaten niemals gesehen. - Immer grenzt eine scharfe Linie den hyalinen Mantel nach aussen ab. Ist die Entartung eine hochgradige, so erscheinen die Follikel als Plaques, welche aus einzelnen hyalinen Scheiben zusammengesetzt sind. Diese Scheiben sind durch ganz schmale Streifen lineär gerichteter Kernreihen von einander getrennt. Offenbar sind an diesen Stellen benachbarte Gefässchen in grösserer 
Anzahl zu gleicher Zeit entartet, und haben durch Compression das zwischenliegende Gewebe zur Atrophie und zum Schwund gebracht, derart, dass sie ǹahezu ganz zusammengerückt sind. Bei diesem Vorgang bleiben schliesslich von dem lymphatischen Parenchym nur noch die Fasern des Reticulums und spärliche Zellen allein übrig.

Studirt man diesen Schwund an grösseren Uebersichtspräparaten, so gewinnt man die Ueberzeugung, dass derselbe von dem Centrum der Follikel aus nach den begrenzenden Lymphsinus zv vorschreitet, so dass ihre äusserste Grenzschicht gegen den Lymphsinus lange Zeit Widerstand leistet. Endlich wird auch diese Grenzschicht ergriffen. Die hyalinen Knäuel wölben sich in die Lumina der Sinus ein, bringen atich diese zur Verödung, und schliesslich tritt an Stelle des Lymphdrüsengewebes ein gleichmässiges Convolut von unförmlichen glasigen Massen, an welchen von Gefässstructur nichts mehr zu erkennen ist. Die Betheiligung des lymphatischen Parenchyms an dem pathologischen Prozesse scheint demnach nur eine passive zu sein. Niemals wird dasselbe, bei ausgebildeten Formen, im Zustande der entzündlichen Reizung getroffen. Ferner kommt es offenbar durch den Schwund des lymphatischen Parenchyms zu einer starken Verkleinerung der Drüsen, deren bereits früher Erwähnung geschah, während in den ersten Stadien, wo die Lymphdrüsensubstanz noch nicht atrophisch ist, aus der Volumzunahme der Gefässe eine Vergrösserung der ganzen Drüsen resultiren dürfte.

Die hyaline Substanz ersebeint fast constant, jedoch in sehr variablem Massa mit feinkörnigen Ablagerungen von Kalksalzen inerustirt. Stets findet die Ablagerung diffus statt. Sie bildet niemals irgendwelche Figuren, wie von Zellen etc. - Beim Ausziehen der Kalksalze durch Säuren bleibt die hyaline Substanz nahezu unverändert zurück. Diese Kalkincrustation scheint das Endstadium des Prozesses zu sein, und dazu zu dienen die pathologische Neubildung zu fixiren.

Die hyalinen Gefässe sind fast sämmtlich kleinste arterielle Aestchen. Vielleicht zeigen doch auch gelegentlich, aber in viel geringerer Frequenz, kleinste Venen diese Veränderung. Dagegen geht von den arteriellen Capillaren die Affection auf die eigentlichen Capillaren in grosser Ausdehnung über.

Eine vorgenommene Prüfung der hyalinen Substanz mit mecbanischen und den üblichen chemischen Agentien ergab Folgendes. 
Beim Zerzupfen zeigt die Substanz einen hohen Grad von Derbbeit und Zähigkeit, beim Zerreissen entstehen scharfe Risslinien, bevor die Gefässe aber reissen, werden sie auf längere Strecken hin aus dem Gewebe, mit welchem sie in ziemlich laxem Zusammenhange stehen, herauspräparirt. Dem Ausschütteln und Auspinseln gegenüber zeigte die Substanz gleichfalls einen nicht geringen Grad von Widerstandsfähigkeit.

Bei Zusatz von Wasser, Kochsalzlösungen, bleibt die Substanz diaphan, glänzend, intensiv lichtbrechend. Sie zeigt keine Quellung. - Durch Jodjodkalium wird sie schwach strohgelb gefärbt. Eine Affinität der Substanz für Jod, ähnlich wie die der amyloiden Substanz, existirt also ganz und gar nicht, -- Aether, Chloroform lassen sie unverändert, - mineralische Säuren wirken aufhellend auf sie ein. Eine Quellung kommt dabei aber kaum zu Stande. Bei Verkalkung ist die Aufhellung natürlich eine sehr intensive. Anwendung von Schwefelsäure liefert noch den Beweis, dass es sich um Kalk handelt, indem sich zahlreiche Gypsnadeln bilden. Ebenso bewirkt Essigsäure nur Aufhellung, nicht Lösung, selbst nicht bei gelindem Kochen. Alle Versuche mit Hülfe von Säuren Kerne oder zellige Bildungen sichtbar zu machen, blieben fruchtlos. - Anwendung von Alkalien bewirkt Aufhellung in extremem Grade, so dass die Substanz absolut amorph, glashell erscheint. Gleichzeitig quillt sie auch ziemlich stark. - Beim Kochen mit Wasser schrumpit die Substanz etwas ein. Sie scheint selbst durch längeres Kochen nicht gelöst zu werden.

Was die üblichen Tinctionen betrifft, so wurde die Substanz intensiv gefärbt durch Carmin, Bikrocarmin, Eosin, gar nicht gefärbt durch Hämatoxylin, Anilinbraun.

Aus dieser Untersuchung lässt sich folgern, dass die byaline Substanz mit der amyloiden, mit welcher sie physikalisch ziemlich grosse Aehnlichkeiten bat, nichts gemein hat. Auch um Anbildung einer Art von sclerotischem Bindegewebe scheint es sich den chemischen Ergebnissen zufolge, nicht zu handeln. Die Substanz scheint den sogenannten colloiden Stoffen verwandt. Mit dem Elastin bat sie vielleicht noch am meisten Gemeinschaft.

Es kam nun darauf an, die Genese dieser hyalinen Substanz nachzuweisen, und womöglich festzustellen, welchem Prozesse sie ihre Entstehung verdanke. Es fand sich auch, wenngleich nur in 
einem Fall, neben hyaliner Entartung der Gefässe noch eine andere bestimmte Veränderung vor. Diese Veränderung wurde aber niemals an ein und derselben Drüse mit hyaliner Entartung zusammen gefunden, wohl aber in anderen Drüsen desselben Falles. Letztere zeigten im Allgemeinen den Habitus, wie er bei Reizung leichten Grades sich gestaltet, einen Zustand mässiger entzündlicher Hyperplasie. In älteren Fällen wurde diese Veränderung niemals gefunden. Im Wesentlichen besteht sie darin, dass einzelne Gefässe eigenthümliche spindelförmige Anschwellungen zeigen, bei schwacher Vergrösserung meist von grobkörnigem Aussehen. Bei starker Vergrösserung erscheinen diese Anschwellungen gebildet aus zelligen Elementen, welche in verschiedener Dicke mit den Gefüssen verklebt und zusammengebacken liegen. Die einzelnen Elemente, mit ziemlich kleinen, runden oder ovalen Kernen versehen, scheinen etwa weissen Blutkörperchen an Grösse gleichzukommen, wenigstens nach der Dichtheit der Kerne zu schliessen; denn die Zellgrenzen sind vollständig verwaschen, und einzelne Zellen wurden aus der Masse nicht isolirt. Es handelt sich aber nicht um eine einfache Auflagerung von Zellen auf die Gefässe, sondern um eine dichte Infiltration der Gefüsshäute, speciell der Adventitia. Denn die Cohärenz der Zellen unter sich und mit den Gefässhäuten ist eine relativ sehr feste. Die verdickten Gefässe lassen sich sammt ihren Verdickungen auf ziemlich weite Strecken aus dem Gewebe reissen. Die Hauptmasse der Verdickungen, nämlich das die Kerne einbettende Protoplasma, ist leicht körnig, diaphan, ziemlich stark lichtbrechend; die Kerne zeigen zarte nicht sehr scharfe Contouren.

ob nun aus diesen Anhäufungen von zelligen Elementen die byaline Substanz hervorgeht, etwa in der Art, dass die Kerne der Zellen schwinden, die einzelnen Protoplasmaklümpchen zusammenfliessen, die Durchsichtigkeit der Substanz steigt, bis endlich die Gefässe amorphe hyaline Schläuche bilden, darüber konnte ein sicherer Beweis nicht geliefert werden. - Ebensowenig liess sich entscheiden, ob man den ganzen Prozèss etwa als einen entzündlichen aufzufassen berechtigt sei.

Es fragt sich nun noch, ob der Prozess primär die Gefässe betrifft, oder ob er aus der Nachbarschaft auf dieselben übergeleitet wird. Das die Gefässe umgebende Iymphatische Gewebe befindet sich aber stets in ziemlich normalem Zustande. Es spricht dieser 
Umstand mit Wahrscheinlichkeit für die Annahme eines primär an den Gefässhäuten seinen Sitz aufschlagenden Prozesses. Derselbe ist jedenfalls chronischer Natur. Die eigenen ihn charakterisirenden Züge beruhen in der eigenthümlichen hyalinen Umwandlung, welcher die von ihm afficirten Gefässe anheimfallen. Schliesslich endet der Prozess mit gänzlicher.Verödung der Drüsen, und Verkalkung der hyalinen Substanz.

Was nun die Aetiologie dieser eigenthümlichen Entartung betrifft, so scheint es nur möglich auf Grund der Vergleichung sämmtlicher bei der Section zusammengefundener pathologischer Momente zu einer Hypothese gelangen zu können. Es mögen desswegen an dieser Stelle die auf das Nothwendige abgekürzten Sectionsprotocolle ihren Platz finden.

Fall 1. Inguinald̊rüsen.

Alte eczematöse Prozesse an den unteren Extremitäten.

Befund: Drüsengefässe in mässigem Grade hyalin entartet. Hochgradige diffuse Verkalkung der Gefässe.

Fall 2. Axillardrüsen, exstirpirt.

Frau von 55 Jahren.

Cystadenoma Mammae.

Weder Herzhypertrophie noch Arteriosclerose an der lebenden Person nachzuweisen.

Befund: Drüsengefässe in sehr geringem Grade hyalin entartet. Geringe diffuse Verkalkung.

Fall 3. Inguinaldrüsen.

Mann von 42 Jahren.

Fractura tibiae vor 10 Jahren, seither Ulcera cruris.

Befund: Drüsengefässe zum Theil hyalin, zum Theil zellig incrustirt. Gar keine Verkalkung.

Fall 4. Inguinaldrüsen.

Mann von 81 Jahrea.

Chronische Plenritis. Marasmus senilis. Decrepidität.

Befund: Drüsengefässe sehr intensir hyalin entartet. Geringe Verkalkung.

Fall 1, à und 4 gingen mir ohne weitere anatomische Details 20.

Fall 5. Lumbale, inguinale, axillare, jugulare, Hals -, maxillare Lymphdrüsen.

Mann von 74 Jahren.

Carcinoma mandibulae. Exstirpation. Aeussere Wunde zum Theil vernarbt. Beiderseitige Schluckpneumonie mit jauchigen Erweichnogsherden.

Befund: Drüsengefässe sehr intensiv hyalin entartet. Sehr wenig Verkalkung. 
Fall 6. Mann von 52 Jahren. Lumbaldrüsen.

Phthisis pulmonum.

Mässige Dilatation und Hypertrophie des rechten Herzens. Venöse Stase. Aorta mässig dilatirt.

Befund: Drüsengefăsse mässig entartet. Gar keine Verkalkung.

Fall 7. Mann von 61 Jahren. Lumbaldrüsen.

Exquisite 0steomalacie. Hochgradiges Lungenemphysem. Dilatation des rechten Herzens, Venöse Stase.

Befund: Drüsengefässe hochgradig byalin entartet. Gar keine Verkalkung? (Chromsäure.)

Fall 8. Frau von 93 Jahren. Lumbaldrüsen.

Senile Osteomalacie. Hochgradiger eitriger Bronchialkatarrh. Randemphysem der Lungen.

Herz beiderseits vergrössert, besonders rechts. Kein Klappenfehler. Hochgradige venöse Stase.

Dedem. Cyanotische Induration sämmtlicher Organe. Ziemlich starke Arteriosclerose. Sclerose der Hirngefässe.

Befund: Drüsengefässe mässig entartet, kaum Verkalkung.

Fall 9. Mann von 74 Jahren. Lumbaldrüsen.

Paralytiker im Anfall verstorben. Starkes Lungenödem. Zahlreiche apoplectische Herdchen und miliare Aneurysmen in den grossen Hirnganglien.

Herz sehr kräftig, besonders ist der linke Ventrikel sehr derb und fest contrahirt. Kein Klappenfehler. Nieren normal.

Die parenchymatösen Organe cyanotisch indurirt.

Aorta ascendens dilatirt, Wand derb, steff. Zahlreiche kleine atheromatöse Herdchen und Ulcerationen in der Aorta abdominalis.

Befund: Drüsengefässe gering entartet. Wenig Verkalkung. Die untersuchten Pia- and Gehirngefässe zeigen nichts Abnormes.

Fall 10. Frau von 58 Jahren. Lumbaldrüsen.

Allgemeiner grossartiger Hydrops.

Die parenchymatösen Organe zeigen bochgradige cyanotische Induration.

Herz beiderseits sehr stark hypertrophisch, links ganz enorm, rechts mässiger. Klappen normal. Nieren normal.

Aorta ganz spärliche atheromatöse Plaques. An den peripherischen Arterien keine Veränderung.

Befund: Drüsengefässe mässig entartet. Starke Verkalkung.

Fall 11. Frau von 70 Jahren. Lumbale, inguinale, Axillardrüsen.

Zahlreiche Narben von varlcösen Ulcerationen.

Chronischer Bronchialkatarrh, Lungenödem, Embolie der Arteria pulmonalis, beider Art. crural.

Herz beiderseits enorm hypertrophisch, besonders aber links, rechts mässiger. Klappen normal. Nieren normal. 
Thromben in allen Herzkammern ausser im linken Vorhof.

Sämmtliche parenchymatöse Organe zeigen cyanotische Induration mässigen Grades.

Aorta stellenweise ganz leicht atberomatös. Gehirngefässe kaum atheromatōs. Befund: Drüsengefăsse hochgradig entartet, äusserst geringe Verkalkung.

Fall 12. Mann von 66 Jahren. Lumbale, Beckenlymphdrüsen.

Carcinoma prostatae, Cystitis, Pyelonephritis.

Bronchitis chronica, Emphysem, Lungenödem.

Herz beiderseits stark hypertrophisch, links enorm, rechts mässig. Klappen normal. Nieren normal.

Aorta nur gering dilatirt. Nur ganz geringe atheromatöse Plaques mehr in Gestalt von Flecken.

Mässige atheromatöse Entartung der Gehirnarterien, auch der Arteria coronaria cordis.

Arteriae crurales und alle grössere peripherische Arterien sehr stark atheromatös.

Stark ausgebildete Varicen.

Befund: Drüsengefässe hochgradig entartet. Verkalkung sehr hochgradig.

Fall 13. Frau von 78 Jahren. Lumbale, inguinale Lymphdrüsen.

Embolia art. pulmonalis. Infarctbildung. Pleuritis haemorrhagica.

Herz beiderseits stark hypertrophisch. Klappen normal. Nieren normal.

Ausgedebnte atberomatöse Entartung der Gefässe.

Befund: Drüsengefässe hochgradig entartet. Verkalkung hochgradig.

Von den Făllen 1, 3 und 4, welche mir, wie bereits erwähnt, ohne genaueres Sectionsprotocoll zugingen, mag bei der folgenden Besprechung abstrahirt werden.

Aus diesen Sectionsergebnissen lässt sich bervorheben das öftere Zusammentreffen der hyalinen Entartung der Lymphdrüsengefässe

1) mit schweren Circulationsstörungen speciell im Sinne einer Erhöhung des Druckes im Gefässsystem;

2) mit hohem Alter, in steigender Frequenz mit zunehmendem Alter von $40-50$ Jahren 1 Fall

$$
\begin{aligned}
& -50-60 \quad-3 \text { Fälle } \\
& -60-70-2 \text { - } \\
& -70-93-6 \text { - }
\end{aligned}
$$

3) mit dyscrasischen Zuständen und tiefen Ernährungsstörungen

$$
\begin{aligned}
& \text { Carcinose . . . . • . . . . } 2 \text { Fälle } \\
& \text { Osteomalacie . . . . . . . . } 2 \text { - } \\
& \text { Phthise . . . . . . . . } 1 \text { Fall } \\
& \text { Chronische Geschwüre, Eczeme etc. } 3 \text { Fälle. }
\end{aligned}
$$


Die Circulationsstörungen waren stets hochgradig. Stets mussten sie starke Druckerböhung in den capillaren und terminalen Gefässramificationen bewirken. Diese Druckerhöhung war in drei Fällen (No. 6, 7, 8) durch venöse Stauung, in fünf Fällen (No. 9, 10, 11, 12 , 13) durch arterielle Congestion bervorgebracht. Letztere fünf Fälle waren eigenthümlicher Weise fünf Fälle von ziemlich reiner linksseitiger Herzhypertrophie ohne Klappenfehler, ohne extreme Gefässsclerose, ohne Nierenleiden, obne accentuirte Plethora universalis. Es verleitete dieser Befund nachzuforschen ob nicht etwa eine weit verbreitete Erkrankung der kleinen Gefässchen vorliege, eine Störung der Circulation bewirkend, als deren Folge die Herzhypertrophie $\mathrm{zu}$ betrachten wäre. Untersuchungen des übrigen Gefässsystems zwangen aber von dieser Hypothese abzustehen. Die Entartung konnte bis jetzt nur an den Lymphdrüsengefässen nachgewiesen werden. Wenn aber die Gefässveränderungen zu gering sind, als dass sie ätiologisch für die Herzhypertrophien sich verwerthen liessen, so lässt sich umgekehrt die unerklärt gelassene Herzhypertrophie wohl als ätiologisches Moment für das Zustandekommen der Gefässveränderung verwerthen. Hochgradige linksseitige Herzbypertrophie bewirkt natürlich sebr grosse Steigerung des Druckes im arteriellen System. Hochgradige venöse Stase bewirkt durch Rückstauung dasselbe. Die Constanz von Druckerhöhungen im Gefässsystem in nahezu allen Fällen, in welchen die hyaline Entartung gefunden wurde, lässt mit grosser Wahrscheinlichkeit diese Druckerhöhung als ein für die Affection ätiologisches Moment auffassen. Warum gerade bei einer das ganze Gefässsystem betreffenden Druckerhöhung nur die Gefässchen der Lymphdrüsen von der hyalinen Affection heimgesucht werden, liesse sich vielleicht aus der besonderen Art ihrer Anordnung in einem lockeren, den Gefässwänden von aussen wenig Unterstätzung gewährenden Gewebe erklären.

Dass aber Druckerhöhung in den Gefässen nicht allein genügt um die hyaline Entartung zu Stande zu bringen, beweisen die Beobachtungen, welche an anderen vier sehr hochgradigen linksseitigen Herzhypertrophien ohne Klappenfehler, und an einer sehr grossen Anzahl von Fällen hochgradiger venöser Stase gemacht wurden, wobei sich die hyaline Entartung nicht vorfand. Es müssen, scheint es, stets noch andere Momente mit im Spiele sein. Als solche Mo- 
mente dürften mit einiger Wahrscheinlichkeit Dyscrasien und Senilität hervorzuheben sein. Was die Dyscrasien betrifft, so lassen sich dieselben sehr wohl in dem gegebenen Falle als den Prozess begünstigende Momente auffassen. Es geben dieselben offenbar Anlass zu einer Reizung mehr auf chemischem Wege. Diese Reizung summirt sich mit dem durch die Druckerhöhung gesetzten mechanischen Reiz. Gerade bei den Lymphdrüsen liegt die Annahme einer solchen Einwirkung sehr nahe, weil ja dieselben bekanntlich sehr häufig erkranken bei dyscrasischen Zuständen der verschiedensten Art. Für sich allein bewirken aber dyscrasische Zustände die byaline Affection nicht, wie sich bei der Untersuchung von sehr zahlreichen Fällen aus dieser Categorie zur Genüge ergab. Ausdrücklich ist hier noch zu bemerken, dass Syphilis niemals konnte nachgewiesen werden. Es fällt also jede Veranlassung weg, die hyaline Entartung als eine luetische zu verdächtigen.

Was nun die Senilität betrifft, so wurde, angesichts der zunebmenden Häufigkeit der Affection mit steigendem Alter, der Verdacht rege, dass der ganze Prozess vielleicht nur eine senile Entartung sein dürfte. Die Untersuchung von Lymphdrüsen bei einer grossen Anzahl von Greisen ergab aber stets negative Resultate. Wider Erwarten liess sich sogar zwischen dem Grade der Verkalkung an der hyalinen Substanz und dem Grade der Ausbildung atheromatöser Veränderungen im Gefässsysteme kein constantes Verbältniss auffinden. Doch zwingt die Thatsache, dass die Affection niemals im Kindesalter, einmal im mittleren, zwölfmal in vorgerücktem Alter gefunden wurde, einen gewissen Grad von Senilität, wo nicht als ätiologisches, so doch als prädisponirendes und adjuvirendes Moment zu betrachten.

Als Schlussresultat lässt sich also nur die eine Hypothese mit einiger Begründung aufstellen, dass es abnorme Druckverhältnisse im Gefässsysteme sind, welche bei dyscrasischen, alten, cachectischen Individuen, die Aetiologie zur hyalinen Entartung der Lymphdrüsengefässe abgeben können.

Bei sorgfältiger Durchforschung der Literatur konnte nichts darüber ausfindig gemacht werden, dass die hyaline Entartung der Lymphdrüsengefässe schon beobachtet worden sei.

Wohl aber fand sich, dass an anderen Organen und Gefäss- 
districten schon seit langer Zeit Gefässentartungen beschrieben und bekannt sind, welche anatomisch wenigstens mit der an den Lymphdrüsengefässen beschriebenen hyalinen Entartung einzelne Punkte gemein haben. Besonders reich an solchen Beobachtungen ist die psychiatrische Literatur. Die bezüglichen Werke sind im Index zusammengestellt.

Die beschriebenen Entarlungen sind theils solche, welche allgemein über den Gefässapparat verbreitet, theils solche, welche nur circumscript vorkommen. Da die hyaline Entartung von mir bis jetzt nur auf die Lymphdrüsengefässe beschränkt beobachtet wurde, so dürfte von den Arbeiten der ersten Categorie abgesehen werden. Die als Periarteritis nodosa von Kussmaul, Maier, P. Meyer, - als Arterio-capillary-fibrosis von Gull und Sutton beschriebenen Veränderungen haben anatomisch, genetisch, ätiologisch mit unserer hyalinen Entartung wenig gemein, und es dürfte wohl in Folge dessen eine weitere Besprechung nutzlos sein. Auch E. Klein's Beobachtung von hyalinen Massen an kleinen Arterien der Milz, Nieren, Darmfollikel, liegt ausserhalb unserer Betrachtung, da es sich dabei 1. um acute Krankheiten (Typhus, Scarlatina), 2. um einen auf die Intima scharf beschränkten Prozess bandelt.

Es bleiben also noch die der psychiatrischen Literatur entnommenen Arbeiten zu besprechen. Dieselben rühren besonders von Wedl, Magnan, Arridt, L. Meyer, Schüle, Lubimoff, Adler, Eppinger, Neelsen her. Sie behandeln sämmtlich die Gefässe der Hirnrinde und der Pia Mater. Es erscheint wohl am zweckmässigsten sie zusammenfassend zu betrachten. Die Entartung ist der Dementia paralytica eigen. In ihrem ersten Stadium zeigen die Gehirn- und Piagefässchen zellige Infiltrationen ihrer Wandungen. Die Zellen verkleben fest unter einander und mit den Gefässen. Ibrer Grösse nach entsprechen sie etwa weissen Blutkörperchen. (Neelsen bält sie für epithelioide. Zellen.) Sie besitzen deutliche Kerne. Durch ihre Anhäufung kommen stellenweise spindelförmige Bildungen zu Tage. Diese sind mehr oder weniger circumseript oder diffus. Was die Abstammung dieser zelligen Elemente betrifft, so theilen sich die Autoren in die zwei Möglichkeiten, theils einer zelligen Infiltration der adventitiellen Răume, theils einer Proliferation der Kerne der Gefässwände, wie 
wenn es nicht bei einem evident als chronisch entzündlich aufzufassenden Prozesse am einfachșten wäre, beides zugleich gelten zu lassen.

Sämmtliche Autoren sind aber ziemlich einig darüber, dass der Prozess nicht auf dieser Stufe stehen bleibt, sondern eine eigenthümliche Metamorphose der infiltrirten Gefässè vor sich geht. Die Zellen vẹsschmelzen enger unter einander. Gleichzeitig werden sie grösser, undeutlich, durchsichtig, stark lichtbrechend. Die Kerne sind bald nur noch mit Hülfe von Reagentien zu Gesicht zu bringen. Dadurch werden die Gefässwandungen verdickt, und bekommen ein glashelles Aussehen. Schliesslich erscheinen sie als dickwandige, glashelle Schläuche, vollständig amorph. An diesen tritt, als Alterssymptom, diffuse Verkalkung auf.

Diese Schilderung hat sowohl anatomisch als auch vielleicht genetisch viel Aehnliches mit dem bei der hyalinen Entartung der Lymphdrüsengefässe beobachteten Vorgang. Die von den Autoren gegebenen chemischen Untersuchungen stimmen gleichfalls bis auf unwesentliche Differenzen mit den eigenen überein. Aetiologisch berufen sich die Autoren auf Reizung der Gefässe durch häufige Congestionen, auf Kreislaufstörungen, auf Senilität. Auch dies sind gemeinschaftliche Gesichtspunkte.

Es handelt sich wahrscheinlich um einen anatomisch sehr nahe verwandten Prozess. $\mathrm{Zu}$ der Annahme einer ldentität der Prozesse liegt aber absolut keine Veranlassung vor. Eine solche müsste vielleicht zugegeben werden, wenn einmal an ein und demselben Individuum gleichzeitig hyaline Entartung der Gehirn- und Lymphdrüsengefässe gefunden würde. Dann könnte die Hypothese für die Discussion reif sein, ob es sich nicht um eine allgemeine Erkrankung des Circulationsapparates handle, welche an den verschiedensten Localitäten zum Ausdruck kommen kann. Bis jetzt konnte aber ein solches gleichzeitjges Vorkommen von mir nicht beobachtet werden. Meinerseits ist àlso jeder Versuch eines Vergleiches verfrüht. Ebenso wenig haben die Psychiater jemals eine Veränderung in den Lymphdrüsen verzeichnet, was allerdings nicht wundern kann, da sie dieselben wabrscheinlich nicht untersucht baben. Dagegen wurde in dem eigenen Fall No. 9, welcher einen alten Paralytiker betraf, wohl die hyaline Entartung der Lymphdrüsengefässe, 
nicht aber die erwartete byaline Entartung der Gehirngefässchen ausfindig gemacht.

Als Schlussresultat obiger Betrachtungen dürfte sich Folgendes über die untersuchte Affection aussagen lassen.

Es giebt eine eigenthümliche Erkrankung kleinster Gefässästchen, welche wesentlich nur an den Lymphdrüsen vorkommt. Dieselbe bestebt in Umbildung der Gefässe zu byalinen Schläuchen, unter starker Verdickung ihrer Wand und Verengerung ihres Lumens. Das hyaline Material entsteht vielleicht aus einem zelligen Infiltrat der Gefässhäute, speciell der Adventitia. Das Ende des Prozesses beruht in diffuser Verkalkung. Seinen chemischen Eigenschaften nach ist der hyaline Stoff von den bindegewebigen Substanzen za trennen, und der Sammelklasse der Colloidstoffe zuzurechnen. Am besten dürfte wohl der Name „Hyaline Entartung“ für den Prozess passen. Als ätiologische Momente des Zustandekommens der Affection dürften besonders Circulationsstörungen anf dyscrasisch-senilem Boden von Belang sein.

\section{B. Hyaline Entartung des eigentlichen Lymphdrüsengewebes.}

Im Verlaufe meiner Untersuchungen über die hyaline Entartung der Lymphdrüsengefässe fand jch zweimal Gelegenheit, eine eigenthümliche hyaline Entartung an den Lymphdrüsen zu beobachten. Dieselbe betraf nicht die Gefässe, sondern speciell das eigentliche Lymphdrüsengewebe. Das hyaline Material zeigte aber mikroskopisch sowohl als chemisch eine ziemlich genaue Uebereinstimmung mit dem bei der hyalinen Gefässentartung beobachteten, und dürfte wohl desshalb diese Affection letzterer hier anzureihen sein. In beiden Fällen war die Affection ausschliesslich auf die Drüsen des Stammes beschränkt. Die peripherischen Drüsen dagegen blieben frei. Beide Fälle betrafen Individues aus dem mittleren Lebensalter, welche unter ziemlich unbestimmten Symptomen, - klinisch dem Bilde des acuten Miliartuberculose noch am meisten entsprechend in ziemlich acuter Weise zu Grunde gegangen waren. Die Section ergab folgende eigenthümliche Verhältnisse: 
Fall 1. P. Valentin, 37 Jahre alt.

Beiderseitige Phthisis Bulbi. Optici atrophisch. Dessgleichen die Tractus optici.

Herz. Endocarditis acuta mitralis. Tuberkelähaliche Knötchen am Endocardium und Myocardium.

Langen durchsprengt mit Knötchen, welche Toberkeln ziemlich ähnlich sind.

Milz, Nieren, Leber mit zablreichen Kaötchen besetzt.

Alle Lymphdrüsen des Stammes sind enorm vergrössert, von zäh elastischer, fester Consistenz.

Die Aorta liegt in grossen Packeten von Drüsen fest eingebacken.

Fall 2. P. Johann. 30 Jahre alt.

Ganz leichtes 0edem der unteren Extremitäten.

In der Bauchhöhle àrei Liter einer stark trühen fibrinösen Flüssigkeit.

Das ganze Peritoneum, sowohl das parietale als das viscerale, besonders aber das Netz zeigen eine continuirliche Eruption von miliaren bis erbsengrossen weisslichen oder gelblichen Knötchen. Dazwischen zahlreiche punkt- und strichförmige Hämorrbaglen. Die Leber und die Milzkapsel allein zeigen relativ wenig solche Knötchen.

An der linken Pleura keine Knötchen. Der Pleurasack enthält etwa 1 Liter gelblicher Flüssigkeit. Rechts ist die Lunge total adbärent. Die Adbäsionen sind durchsetzt mit miliaren bis erbsengrossen Knötchen. Dieselben zeigen eine gelbweisse Farbe und auf dem Schnitt eine eigenthümlich glatte Fläche.

Beide Lungen zeigen in der Spitze einige ganz kleine, glatt ausgekleidete Cavernen. Das ganze Lungenparenchym ist durchsprengt mit Knötchen von gelblicher Farbe, von fester Consistenz. Die mediastinalen Lymphdrüsen sind ausserordentlich vergrössert, von zäh elastischer, fester Consistenz.

Herz schlaff, blass, klein und welk.

Die Milz ist etwas vergrössert, schlaff, blutreich. Sie zeigt eine grosse Anzahl von bis nahezu kirschgrossen Herdchen derselben Farbe und Consistenz wie die mediastinalen Drüsen.

Nteren normal.

Leber anf der Oberflăche und anf dem Schnitt spärliche Herdchen beschriebener Beschaffenheit.

Die Lymphdrüsen des Beckens und der Banchböhle sind enorm, bis zu apfelgrossen Packeten, welche die Aorta fest einbacken, vergrössert. Sie zeigen alle die beschriebene Beschaffenheit.

Das Mesenterinm, das Netz, die Gedärme sind continuirlich mit Knötchen übersäet, mit Ausnahme des Coecum.

Die Hoden zeigen auf dem Schnitt eine grosse Anzahl von Herdchen, welche dieselben Eigenschaften zeigen.

Die specielle Untersuchung der Lymphdrüsen ergab nun Folgendes. Die Consistenz ist eine zäh elastische. Die Farbe äusserst blass, bis rein weiss. Die Grösse erreicht bei einer gewissen An- 
zahl, besonders solcher, die an der Aorta gelegen sind, nahezu die einer grossen Kastanie. Auf dem Schnitt zeigen die Drüsen ein eigenthlimliches Bild. Die Schnittfläche ist absolut glatt und eben. Die Substanz quillt nicht hervor, sinkt auch nicht zurück. Sie ist trocken, sehr fest, zähe, der Consistenz nach am besten, abgesehen von der Brüchigkeit, mit amyloider Leber zu verolejehen. Die Consistenz ist an allen Stellen eine gleichmässige; weiche oder käsige Stellen sind nicht ausfindig zu machen. Die Farbe ist weissgrau bis absolut weiss. Das Gewebe scheint ausserordentlich anämisch. Nur hier und da erscheinen zerstreute, röthliche Zeichnungen in der weissen Substanz. In dem weissen Gewebe erscheinen dann unregelmässig zerstreute Herde einer ganz diaphanen glasigen Substanz. Von der normalen Zeichnung, von Follikeln, Rinden- und Marksubstanz tritt nichts mehr zu Gesicht. Die Drüsenkapsel ist meist verdickt, blass, und zeigt eine geringe Vascularisation. Neben diesen vollständig entarteten Drüsen findet man noch in äusserst spärlicher Anzahl andere, welche in einem ziemlich normalen adenoiden Gewebe eingebettet, unregelmässig contourirte Knötchen zeigen, welche auf dem Schnitt dieselbe hyaline Beschaffenheit zeigen, wie sie an den intensiv betroffenen Drüsen beobacbtet wird.

Bei der mikroskopischen Untersuchung zeigt sich nun alsbald, dass in den meisten Drüsen von einer normalen Drüsenstructur keine Spur mehr vorhanden ist. An deren Stelle erscheint ein ganz eigenthümlicher Complex von Substanzen und Geweben, die zu rubriciren beim ersten Blick ganz unmöglich erscheint. Zellenreiche, zellenarme und zelliger Elemente gänzlich entbehrende Stellen wechseln in buntem Gemisch mit einander ab. Je höher der Grad der Entartung, desto befremdender wird der Anblick. Auffallend ist dazu noch die Gleichmässigkeit der Entartung an den afficirten Drüsen, und die grosse Extensität der Affection, welche nur äusserst spärliche Drüsen relativ verschont lässt. Die Seltenheit solcher jugendlicher Stadien der Entartung, an welchen der Hergang des Prozesses am leichtesten zu verfolgen wäre, erschwerte die Untersuchung nicht unerheblich. An den stark betroffenen Drüsen war über die Art des Prozesses gar nichts mehr heraus zu bringen. Man konnte stellenweise daran zweifeln, dass man es mit Lymphdrüsen zu thun habe. Es kostete daher ziemlich viel Zeit und 
Mühe, nur eine Hypothese über die Genese des Prozesses aus zahlreichen einzelnen Beobachtungen zu construiren. Inwiefern dieselbe der Wirklichkeit nahe tritt, möge dahin gestellt bleiben.

Es erscheint zweckmässig, die Schilderung der verschiedenen Typen der vorhandenen Gewebe mit ihren Uebergangsformen, da sie wahrscheinlich doch nur verschiedene Altersstufen ein und desselben Prozesses sind, mit der Schilderung dieses muthmasslichen Prozesses zu verflechten. Auf diese Art scheint die Schilderung an Klarheit gewinnen zu dürfen.

Untersucht man eine der wenigen Drüsen, welche noch ein ziemlich normales Aussehen bieten, so findet man an ibnen etwa folgende Veränderungen. Die Drüsen sind etwas vergrössert. Follicularsubstanz und Lymphwege sind an innen noch ziemlich leicht zu differenziren. Die Lymphbahnen zejgen gewöhnlich die Verbältnisse des von den Autoren als Katarrh bezeichneten Vorganges, nämlich Anhäufung von zablreichen grossen epithelioiden Zellen, vermischt mit zahlreichen Lymphzellen, und etwas Quellung ibrer Spannfasern. Die Follicularsubstanz zeigt ebenfalls Symptome eines mässigen irritativen Vorganges. Das Reticulum ist lejcht gescbwollen, die Lymphzellen scheinen vermehrt, klein, etwas protoplasmaarm. Zahlreiche grosse protoplasmareiche, oval gekernte epithelioide Zellen liegen ohne jede Ordnung im adenoiden Gewebe zerstreut. An den Gefässen und Gefässscheiden tritt nichts Besonderes zu Tage. Die Drüsenkapsel erscheint etwas aufgelockert, zellenreich, kurz man erhält ganz das Bild, welches man häufig zu beobachten Selegenheit hat, wenn man geschwollene, von sogenannten scrofuJösen Subjecten exstirpirte Lymphdrüsen untersucht. Nach und nach wird aber die Differenzirung zwischen Follicularsubstanz und Lymphbabnen undeutlich und verwaschen. Die Lymphbahnen werden mit epithelioiden und Lymphzellen nabezu ganz angefüllt, in der Follicularsubstanz nehmen die epithelioiden Zellen ebenfalls $z u$, so dass sehliesslich auf dem Schnitt das Gewebe ein ziemlich gleichmässiges wird. Auch das Reticulum quilt, zeigt grosse Kerne und breite undeutlich begrenzte Bälkchen. $\mathrm{Zu}$ dieser Zeit schien es gelegentlich, als käme hie und da eine eigenthümliche Entartung an zelligen Elementen zu Stande.

Es schien nämlich, als unterläge der protoplasmatische Leib gewisser Zellen einer substantiellen Veränderung zu einem hỵalinen 
Material, unter Zunahme der Grösse, des Glanzes und des Lichtbreehungsvermögens des Zellenleibes. Gleichzeitig mit diesen glasig hyalinen Zellen kamen auch solche vor, welche sich durch den Mangel eines Kernes bemerkbar machten. Gestalt und physikalische Merkmale dieser amorphen Klümpchen erregten aber die Vermuthung, dass es sich um das vom Kerne befreite Protoplasma der eben beschriebenen Zellen bandle. Eine Blähung oder Auflösung von Kernen wurde niemals bemerkt, wohl aber kamen gelegentlich solche Zellen vor, deren Kerne ganz an die Peripherie gerückt lagen; auch schien es gelegentlich als ständen solche Klümpchen mit einem nackten Kerne durch einen ausgezogenen Protoplasmafaden in Verbindung. Es liesse sich also vielleicht eine Trennung von Zellprotoplasma und Zellkern als Ursprung der byalinen Substanz ansehen. Wie dem auch sei, nach und nach entstehen unter Vermehrung und gegenseitiger Abplattung der Klümpchen mehr oder weniger abgegrenzte Haufen. Die einzelnen Klümpchen, welche die Haufen byaliner Substanz zusammensetzen, bleiben aber oft wie durch Sprünge und spaltförmige Lücken von einander getrennt. In diesen Lücken liegen nackte Kerne in wechselnder Anzahl. Die Klümpchen sind stets an der Peripherie der Haufen am deutlichsten. Im Centrum der Haufen, gelegentlich auch einmal zuerst an der Peripherie, fliessen die Klümpchen, unter stetiger Zunahme ihrer Durchsichtigkeit, zu einer homogenen structurlosen Substanz zusammen. Die Form der Haufen scheint von den Fasern des Reticulums, in welche die hyaline Substanz eingegossen liegt, bedingt zu werden. - Es kann nur schwer entschieden werden, wie das Verhalten der Capillargefässchen in den Haulen sich gestaltet. Die kleineren scheinen unterzugehen, die grösseren dagegen bleiben stets offen und wegsam. - Da diese hyalinen Klümpchen so zahlreich und dicht gedrängt liegen, und ihre hyaline Substanz das Licht so auffallend stark bricht, so resultirt hieraus die für das blosse Ange weiss erscheinende Farbe der ganzen Substanz, so wie ihre Undurchsichtigkeit, obwohl jeder einzelne Klumpen mikroskopisch vollständig glasig byalin ist. - Erst wenn der höchste Grad der Entartung der Druisen erreicht ist, wenn nämlich fast nur noch hyaline Substanz existirt, ist auch makroskopisch das ganze Drüsengewebe homogen und in hohem Grade durchsichtig geworden, ähnlich wie amyloides Gewebe. 
In diesem Stadium ist mikroskopisch überhaupt von Lymphdrüsenbau nichts mehr zu erkennen; durchweg besteht das Gewebe aus Klumpen einer glasig hyalinen Substanz, welche die verschiedensten Formen besitzen, und keine Kerne enthalten. Während in den jüngeren Stadien sich an den Klumpen noch gelegentlich Andeutungen einer mosaikartigen Zusammensetzung aus einzelnen byalinen Elementen zeigen, sind solche jetzt nicht mehr daran wabrzumehmen. Auf dem Schnitt zerfällt die hyaline Substanz in ovale oder stumpfeckige Platten. Dieselben sind eingefasst in ein Maschenwerk von zablreichen gröberen und feineren verästelten Bälkchen. Diese Bälkchen sind meist feste Streifen einer ganz zart längsfibrillirten, Kerne fübrenden Substanz. Die Kerne sind stellenweise zahlreich, stets aber klein und kümmerlich. Die ganze Anordnung der Bälkchen entspricht vollkommen der Anordnung des normalen Drüsenreticulums, in dessen erweiterten und gedehnten Maschen die hyalinen Klumpen fest eingegossen liegen. Was die zahlreichen an den Bälkchen sitzenden Kernchen für eine Bedentung haben, ob sie etwa, nach der erwähnten Hypothese, deu bei der hyalinen Eutartung von den Zellen ausgestossenen Kernen, oder atrophirtem Lymphgewebe entsprechen, lässt sich nicht mit Sicherheit entscheiden.

Was das Verhalten gegen chemische Agentien betrifft, so ergaben sich zwischen der klumpigen und der ganz gleichmässigen byalinen Substanz einige Differenzen. Mineralsäuren, Essigsäure, Alkalien wirken auf beide Substanzen aufhellend, nicht lösend ein. Durch Kochen und Maceration wird die klumpige Substanz etwas körnig getrübt und schrumpft ganz leicht, die gleichmässige Substanz bleibt unverändert. Jodjodkalium bewirkt keine Färbung. Bei Anwendung des Millon'schen Reagens wird die klumpige Substanz rosigroth, die gleichmässige nicht gefärbt. Durch Carmin wird die klumpige Substanz diffus intensiv roth, die gleichmässige leicht hellrosa gefärbt. Hämatoxylin und Anilinbraun färben die klumpige Substanz leicht und diffus (das Hämatoxylin sehr launenhaft), lassen dagegen die gleichmässige Substanz vollständig ungefärbt.

Diese Eigenschaften scheinen für eine substantielle chemische Umwandlung des hyalinen Materials zu sprechen. Die klumpige Substanz entspricht am meisten den protoplasmatischen Eiweissstoffen. Die hyaline Sustanz zeigt eine eigenthümliche Ueberein- 
stimmung mit derjenigen, welche früher als nur an den Lymphdrüsengefässen vorkommend beschrieben wurde.

Summirt man sämmtliche Befunde zusammen, so scheint es sich um einen Prozess zu handeln, den man als einen subacut oder chronisch entzündlichen, ein eigenthümliches Materjal producirenden aufzufassen wohl berechtigt ist. Diese Auffassung bestätigte auch die Untersuchung der an den parenchymatösen Organen vorhandenen Knötchen. Es fand sich nämlich, dass es sich meist um kleine Entzündungsherdchen bandle, chronisch interstitieller Natur. Stets trat aber in allen Organen, als gemeinschaftlicher Zug, die eigenthümliche Umwandlung der Knötchen zu einem homogenen, in älteren Fällen mehr oder weniger hyalinen Material ein. Echtes käsiges Material, echte Riesenzellen kamen deutlich nicht zu Gesicht. Selbst die miliare Eruption von Knötchen am Peritoneum in Fall 2 zeigte schon in den miliaren Herdchen Tendenz zur Production einer eigenthümlichen Substanz. Nach welchen präexistirenden Apparaten die Ablagerung zu Stande kommt, liess sich auch da nicht genau eruiren.

Es bleibt nun noch die Frage aufzuwerfen, wie der ganze Prozess eigentlich zu rubriciren sei. Klinisch fällt derselbe ohne Zweifel unter die Rubrik Tuberculose. Als solche wurde die Affection während des Lebens an den Patienten ibrem Verlaufe nach diagnosticirt. Anatomisch ist die Frage eine complicirtere. Es fragt sich, ob man die Knötchen anatomisch als Tuberkel auffassen darf, oder nicht. Betrachtet man den Tuberkel überhaupt als eine gefässlose lymphomähnliche Bildung mit regelmässigem Ausgang in Verkäsung, so wird es schwer die in den parenchymatösen Organen gefundenen Knötchen Tuberkel zu nennen. Fasst man aber speciell die Lymphdrüsen in's Auge, folgt man der neueren, in Schüppel's umfassender Monographie aufgestellten Definition, so muss man gänzlich darauf verzichten den vorliegenden Prozess als Tuberculose zu bezeichnen. Von dem von Schüppel als charakteristisch aufgestellten typisehen feineren Bau wurde niemals etwas wahrgenommen. Besonders aber wurde keine echte Riesenzelle gesehen, dasjenige Gebilde, welches Schüppel als das constanteste bezeichnet. Endlich wurde niemals eine echte käsige Metamorpbose beobachtet. 
In Fall 40, S. 75 seines Werkes beschreibt $S c b u ̈ p p e l$ eine Affection der Lymphdrüsen, welche möglicherweise mit dem vorliegenden Prozesse etwas gemein haben dürfte. Es handelt sich um die Drüsen eines 19 jährigen Mädchens, welches ziemlich acut an einer allgemeinen Miliartuberculose verstarb. Die eigenthümliche Entartung beschreibt Schüppel unter dem Namen Verhornung. Es fanden sich nämlich in keineswegs scharf umschriebenen Herdchen, welche epithelioide Zellen enthielten, solche Zellen vor, welche unter Verlust ihrer Kerne zu homogenen stark glänzenden Kugeln sich umwandelten, dann unter sich und mit dem Reticulum zu einer gleichıässig zusammenhängenden homogenen Substanz zusammenschmolzen. Später fand keine Verkäsung statt. Schüppel erklärt die auf diese Weise zu Stande gekommene Substanz als bindegewebiger Natur. Einiges in diesem Befunde erinnert lebhaft an die eigenen Bilder, Anderes ist abweichend. Da nähere Details nicht mitgetheilt sind, so muss von einem genaueren Vergleiche der Fälle abgesehen werden.

Demnach läge bis auf Weiteres keine Veranlassung vor den Prozess als Tuberculose aufzufassen. Derselbe scheint ein herdweise auftretender chronisch entzündlicher Prozess zu sein, mit Bildung eines eigenartigen hyalinen Materials. Jedenfalls ist er, da er, ähnlich der Tuberculose, multipel auftritt, als ein bösartiger zu bezeichnen. Forseht man nun nach dem wahrscheinlichen Ausgangspunkte der Affection, so zeigt sich bald, dass man es offenbar mit einer primären Erkrankung des Lymphdrüsenapparates zu thun hat. Die Lymphdrüsen allein sind hochgradig entartet, in einer Ausdehnung und Form, welche zur Annahme eines bereits langen Bestehens der Affection zwingen, während alle übrigen als Metastasen zu betrachtende Knötchen nur kleine Herdchen und relativ niedrige Stufen des Prozesses zeigen.

Was die Aetiologie des Prozesses betrifft, so dürfte man vorläufig wohl nichts Anderes zu beschuldigen haben, als allgemeine dyscrasisebe Verbälinisse. Ganz dunkel bleibt ebenfalls die Art, auf welche die Metastasen der Affection zu Stande kommen. Eine Verschleppung von Substanzpartikelchen ist bei der Festigkeit und Consistenz der Substanz schwer zu denken. Ueber einen etwaigen 
schädlichen Stoff in gelöster Form lässt sich natürlich nichts Weiteres aussagen.

Als die Arbeit bereits zum Abschlusse gekommen war, erhielt ich Kenntniss von einem Aufsatze von Cornil über Lymphdrüsenentartungen. Demselben liessen sich folgende Punkte entnehmen. In Drüsen, welche Verfasser als tuberculös bezeichnet (Tuberkel $=$ circumscripte nicht scharf begrenzte Infiltration mit Rundzellen), liessen sich Herdchen nachweisen, im Durchschnitt 0,2 Mm. gross, aus glänzenden kernlosen Lymphzellen bestehend, welche durch $\mathrm{Zu}$ sammenfliessen, in die Mäschen des Reticulums eingegossene, hyaline Kugeln bildeten. Diese Gebilde nennt Cornil „Tubercules collö̈des". Es handelt sich in dieser Beobachtung möglicherweise um die jüngsten Anfangsstadien der von mir bescbriebenen hyalinen Entartung. Ich batte selbst Gelegenheit in einem Falle, an vom Halse eines 12 jährigen Mädchens exstirpirten Drüsen ähnliche Gebilde zu beobachten wie sie Cornil beschreibt, konnte aber über ihre Genese nicht zur Entscheidung kommen. Ob aber diese kleiuen colloiden Herdchen, wie sie Cornil nenut, der Anfang zu einer späteren allgemeinen hyalinen Drüsenentartung sein dürten, lässt sich thatsächlich nicht von der Hand weisen, ebensowenig aber verfechten. Die Abbildungen, welche Cornil seiner Abhandlung beigefügt hat, stimmen nabezu in allen Punkten mit den eigenen Objecten überein. Dass es sich um eine allgemeinere Entartung handeln dürfte, spricht Cornil nicht aus.

Von der byalinen Entartung dürte wohl, wie auch Schüppel und Cornil angeben, eine eigenthümliche Entartung zu trennen sein, welche nach abgelaufenen chronischen Entzündungen an den Lymphdrüsen Platz greift. Das Bindegewebe des Reticulums wandelt sich dabei zu einem eigenthümlich sclerotischen zellenarmen Gewebe um, ganz einer eigentlichen Bildung von Narbengewebe entsprechend. Es kann durch dies Gewebe totale Verödung der Drüsen eintreten, welche dann ebenfalls derb und durchsichtig scheinen, bei einiger Aufmerksamkeit aber die faserige Textur ihres Gewebes leicht erkennen lassen. 


\section{L i t e r a t u r.}

Kussmaul und Maỉer, Ueber eine bisher nichtbeschrlebene eigenthümliche Arterienerkrankung (Periarteritis nodosa) in Deutsches Archiv für klin. Med. Bd.1. 1866. S. $484 \mathrm{ff}$.

P. Meyer, Ueber Periarteritis nodosa, in diesem Archiv Bd. 74, Hft. 3, S. $277 \mathrm{ff}$. 1878.

W. GuIl and H, Sutton, On the Pathology of Brights Disease (arterio-capillary fibrosis), in Med. chirurg. Transactions. Vol. 55, p. 273 ff. 1872.

Atkins Aingrose, 0n arterio-capillary fibrosis, in British med. Journal. 3. April 1875 .

E. Klein, Report on Enteric or Typhoid Fever, in Reports of the medical Officer of the privy Council and local Government Board. New Series No. 6. London 1875. p. 80 fif.

E. Klein, Report on the minute Anatomy of Scarlatina. Ibidem. New Series No. 8. London 1876. p. 24 ff.

Wedl, Beiträge zur Pathol. der Blutgef. Colloide Ablagerungen. Wiener acad. Sitzungsber. Bd. 48. 1863. S. $386 \mathrm{ff}$.

Wedl, Histol. Unters. über Hirntheile dreier Idioten. Wiener medic. Jabrbücher 1863. S. $139 \mathrm{ff}$.

Magnan, De la lésion anatomique de Ja Paralysie générale. Thèse de Parls. 1866.

Arndt, R., Eine eigentb. Entartung der Hirngef. Dieses Archiv Bd, 41. 1867. S. $461 \mathrm{ff}$.

Meyer, L., Die Veränderungen des Gehirns in der allgem. progr. Paralyse der Irren, in Medic. Centralblatt 1867, No. 8.

Schüle, H., Beitrăge zur Pathol. und pathol. Histologie des Gehirns und Rückenmarks, in Allgem. Zeitschrift für Psychiatrie 1868. \$. $449 \mathrm{ff}$.

Magnan, De la dégénerescence colloïde du cerveau dans la paralysie générale, in Arch. de physiologie norm. et pathol. T. 2. p. 251 ff. 1869.

0 bersteiner, H., Zur path. Anatomie der paralyt. Geisteskrankh. Dieses Archiv Bd. 52. 1871. S. 510 if.

Meyer, L., Die pathol. Anat. der Dementia paralytica. Dieses Archiv Bd. 58. 1873. S. $270 \mathrm{ff}$.

Lubimoff, A., Studien über die Veränderungen des geweblichen Gehtrnbaues bei der progr. Paralyse der Irren. Dieses Archiv Bd. 57. 1873. S. 371 ff.

Lubimoff, A., Beiträge zur path. Anatomie der aligem. progr. Paralyse, und Mittheilungen über eine besondere colloidartige Degener. der Hirngefässe. Archiv für Psychiatrie 1874. S. $579 \mathrm{ff}$.

Sebüle, H., Sectionsergebnisse an Geisteskranken. Leipzig 1874.

Adler, Ueber einige pathol. Veränderungen an den Hirngefässen Geisteskranker. Archiv für Psychiatrie 1874. S. 77 ff.

Adler, Ueber einige pathol. Veränderungen im Gebirne Geisteskranker. Ibidem S. $346 \mathrm{ff}$. 
Epplnger, Mittheilungen aus dem path. Institute zu Prag, in Vierteljahrsschrift für pract. Heilk. der Facultät Prag. 1875.

Neelsen, Ueber eine eigenthümliche Degeneration der Hirncapillaren. Arch. der

Heilkunde 1876. S. $119 \mathrm{ff}$.

Billroth, Beiträge zur pathol. Histologie. Berlin 1858. S. 125 ff.

W. Müller, Zur Kenntniss des Baues gesunder and krankhaft veränderter Lymphdrüsen, in Zeitschr. für rat. Medicin. 3. Reihe. Bd. 20. 1863. S. 119 ff.

Sch üppel, Untersuchungen über Lymphdrüsentuberculose. Tübingen 1871.

Cornll, Des altérations anatomiques des ganglions lymphatiques, in Journal de l'anat. et de la physiologie. 1878. p. $358 \mathrm{ff}$.

\section{Erklärung der Abbildungen.}

Tafel I.

Fig. 1. a Lymphbahnen (das Detail nicht weiter ausgefúhrt). b Lymphgewebe (das Detail nicht weiter ausgeführt. Schattirte Partien). c Hyalin entartete Gefässnetze. d Normale Gefässe zum Vergleich daneben gezeichnet: Vergr. Hartnack 0c. 3, 0bj. 4 .

Fig. 2. a Hyalin entartetes Gefäss. b Ein solches anf dem Querschnitt. c Grosse byaline Masse, Gefässknäuel. Vergr. H. 0 c. 3, 0 bj. 4 .

Fig. 3. a Hyalin entartetes Gefäss. b Ein solches auf dem Querschnitt. c Ein solches mit Kalkkörnern incrustirt. d Mit Zellen infiltrirtes Gefäss. Vergr H. 0c. 3, 0 bj. 7 .

Fig. 4. a Epithelioide Zellen. b Normale Lymphzellen. c Hyaline Klümpchen. Vergr. H. 0c. 3, 0bj. 7.

Fig. 5. a Protoplasmaklümpchen mit einem Kerne in Verbindung. b Ein hyalines Klümpchen in elner Masche des Reticulums liegend. Vergr. H. Oc. 3, Obj. 7.

Fig. 6. Ein Haufen klumpiger Substanz. Vergr. H. 0c. 3, 0bj.7.

Fig. 7. a Hyaline Klumpen. b Faserzüge mit Kernen und Zellen. c Epithelioide Zellen. d Gefässquerschnitt. Vergr. H. Oc. 3, 0bj. 4 .

Fig. 8. a Hyaline Klumpen von geringen Dlmensionen. b Hyaline Klumpen mit Andeutungen einer mosaikartigen Zusammensetzung. Vergr. H. Oc. 3, Obj. 7. 